This is an Open Access article licensed under the terms of the Creative Commons

\title{
Irinotecan-Induced Dysarthria
}

\author{
Albertine J. Dressel ${ }^{\mathrm{a}} \quad$ Johannes C. van der Mijn ${ }^{\mathrm{a}}$ \\ IJke J. Aalders ${ }^{b}$ Rico N.P.M. Rinkel ${ }^{b}$ \\ Hans J. van der Vliet ${ }^{\mathrm{a}}$ \\ Departments of a Medical Oncology, and ${ }^{b}$ Otolaryngology/Head and Neck \\ Surgery, VU University Medical Center, Amsterdam, The Netherlands
}

\section{Key Words}

Dysarthria $\cdot$ Irinotecan $\cdot$ Colorectal cancer

\begin{abstract}
Colorectal carcinomas are among the most common tumor types and are generally treated with palliative chemotherapy in case of metastatic disease. Here, we describe the case of a 46-year-old patient with metastatic rectal carcinoma who received second-line therapy with irinotecan and developed isolated transient dysarthria (with normal MR imaging of the brain) following each administration of irinotecan. Neurological and logopedical evaluation revealed that the dysarthria predominantly resulted from a reduced capacity in fine-tuning of motor functions of the tip of the tongue and a minimal reduction in the power of speech at labiodental contact. As hypoglossal nerve activity has been reported to be especially susceptible to cholinergic stimulation and irinotecan can cause cholinergic side effects by binding to and inactivating acetylcholinesterase, we suspect this mechanism to be responsible for irinotecan-induced dysarthria.
\end{abstract}

\section{Introduction}

In the US, colorectal cancer is diagnosed in approximately 150,000 patients each year. It is the fourth most common form of cancer in the US and the third leading cause of cancer-related death in the Western world. In patients with localized disease, standard treatment includes surgical resection of the tumor, followed by adjuvant chemotherapy in case of regional lymph node metastases. Approximately $50 \%$ of patients develop distant metastases $[1,2]$. Though curative treatment options are increasingly available in case of metastatic disease, treatment in this situation is still most often palliative. In case of metastatic disease, median overall survival without chemotherapy is approximately 8 months, with a 1 -year survival of $34 \%$. When 5 fluorouracil/leucovorin (5-FU/LV) or the equally effective and toxic oral 5-FU prodrug capecitabine is combined with irinotecan or oxaliplatin and bevacizumab, the median 
survival increases to more than 20 months, with a 1-year survival of 74\% [2-6]. Following first-line treatment with Folfox or Capox, irinotecan is frequently used as second-line therapy. Irinotecan (CPT-11) is a prodrug that requires activation by carboxylesterases to yield the potent topoisomerase I inhibitor SN-38 that inhibits DNA double strand replication and cell division. Well-known side effects of irinotecan are the result of the induction of an acute cholinergic syndrome and include rhinitis, increased salivation, miosis, lacrimation, diaphoresis, flushing, and intestinal hyperperistalsis. This cholinergic activity is caused by binding of irinotecan to the active site gorge of acetylcholinesterase resulting in its subsequent functional inhibition [7]. Here, we describe the case of a patient with metastatic rectal carcinoma who received second-line therapy with irinotecan and developed isolated transient dysarthria following each administration of irinotecan. Although we are aware of the reports of 7 patients experiencing this unique and isolated toxic effect, our case is the 1st to include online supplementary video and speech recordings (www.karger.com/doi/10.1159/000336156) [8-13].

\section{Case Report}

A 46-year-old male was referred to our outpatient clinic because of metastatic rectal cancer. One year earlier he was treated with neoadjuvant capecitabine-based chemoradiotherapy and a low anterior resection in another hospital. Six months later, he was treated with radiofrequency ablation and transarterial chemoembolization because of liver metastases. As this procedure was complicated by sepsis, ischemia of the left liver lobe and abscesses, his clinical condition at that time impeded the use of adjuvant chemotherapy. Unfortunately, new liver metastases, lymph node metastases, and an adrenal metastasis developed over the next months. After referral to our department, first-line palliative chemotherapy (3-weekly: i.v. oxaliplatin $130 \mathrm{mg} / \mathrm{m}^{2}$ day 1 ; oral capecitabine $1,000 \mathrm{mg} / \mathrm{m}^{2}$ b.i.d. days 1-14) was initiated. Treatment was well tolerated and resulted in a partial response. Unfortunately, progressive disease developed after a total of 10 cycles. After a therapy-free interval of 2 months, second-line chemotherapy with irinotecan (i.v. 3-weekly; $350 \mathrm{mg} / \mathrm{m}^{2}$ ) was started. Standard premedication included atropine $(0.25 \mathrm{mg}$ s.c.), dexamethasone (10 $\mathrm{mg}$ i.v.) and ondansetron (8 $\mathrm{mg}$ i.v.). Approximately $45 \mathrm{~min}$ after the irinotecan infusion was started, the patient developed dysarthria. A neurologist confirmed that the dysarthria, which resolved spontaneously within $24 \mathrm{~h}$, was not accompanied by any other neurological symptom apart from the known NCI-CTC grade 1 peripheral polyneuropathy that resulted from prior oxaliplatin exposure. MR imaging of the brain, including diffusion weighted imaging, did not show any structural or perfusion abnormalities (fig. 1). Upon each 3-weekly readministration of irinotecan, the isolated dysarthria recurred and resolved spontaneously within several hours (see accompanying suppl. video). As ondansetron and dexamethasone had also been used previously in combination with first-line chemotherapy in this patient, these agents could not be responsible for the observed dysarthria. A causative role of atropine was excluded by administering atropine premedication ( $0.25 \mathrm{mg}$ s.c.) $2 \mathrm{~h}$ prior to the administration of irinotecan; dysarthria did not occur after atropine alone, while subsequent administration of irinotecan again induced dysarthria. Acoustic analyses (see video and speech recordings) performed by an experienced speech therapist and otolaryngologist indicated a reduced capacity in fine-tuning of motor functions of the tip of the tongue and a minimal reduction in the power of speech at labiodental contact. Also, a reduction in sound coordination was found predominantly for consonants generated by the tip of the tongue. For example, notable changes were observed in the cluster /str/ in stretch, destroy, and stranger requiring a coordinated activity of the tip of the tongue and in practice requiring a rapid relatively large movement of the tongue. The patient experienced the dysarthria as only slightly bothersome and, apart from alopecia, he experienced no other toxicity. After an initial radiological response, treatment with irinotecan was stopped after 9 cycles because of progressive disease (fig. 2). Presently, the patient is successfully being treated with the EGFR inhibitor panitumumab. 


\section{Discussion}

Speech is based on the fast and varied contractions of oral-, pharyngeal-, and laryngeal muscles. Dysarthria is caused by a motility disorder of these muscles caused by upper motor neuron lesions of the cerebral hemispheres or lower motor neuron lesions of the brain stem, but can also result from disruption of the integrated action of upper motor neurons, basal ganglia, and cerebellum [14]. Our patient was evaluated before and during several episodes of irinotecan-induced dysarthria. No other neurological signs or symptoms were noted and radiological evaluation (conventional and diffusion-weighted MRI) showed no abnormalities, which is in line with previously reported cases. Analyses by an otolaryngologist and speech therapist indicated that the dysarthria predominantly affected consonants generated by the tip of the tongue, and although the anatomical level of these disturbances cannot be determined with certainty, it is believed to originate centrally at the level of the brainstem.

The exact pathogenesis of irinotecan-induced dysarthria is still unknown, but several possible mechanisms have been put forward. Gomez et al. [8] reported a case in which tremors and ataxia preceded the development of dysarthria during the third and subsequent cycle of an irinotecan-containing chemotherapy regimen and suggested that the use of atropine could prevent progression to dysarthria. Notably, however, this preventive effect of additionally administered atropine was not observed in our case or in the case reported by Hamberg [12]. This difference could be related to differences in the dose of irinotecan, which was substantially lower in the case reported by Gomez et al. [8] $\left(60 \mathrm{mg} / \mathrm{m}^{2} \mathrm{vs} .350 \mathrm{mg} / \mathrm{m}^{2}\right)$. Atropine itself was not responsible for the observed toxicity as we found that its sole administration to the patient did not result in dysarthria. Because an earlier study demonstrated that the pharmacokinetics of irinotecan were not different in patients developing dysarthria, the isolated dysarthria cannot be explained by differences in systemic clearance of irinotecan [12]. Irinotecan increases cholinergic activity by binding to the active site gorge of acetylcholinesterase resulting in its functional inhibition [7]. Because of its innervation of tongue muscles, the hypoglossal nerve plays an important role in speech and its overstimulation or damage can cause dysarthria. As the density of cholinergic receptors is relatively high in brainstem nuclei and the application of cholinergic agents results in a large increase in hypoglossal nerve activity compared to e.g. phrenic nerve activity $[15,16]$, we believe that the occurrence of isolated dysarthria in patients treated with irinotecan could be related to this apparent intrinsic increased sensitivity of hypoglossal nerve activity to cholinergic stimulation. Unfortunately, we could not directly test this hypothesis because of progressive disease in our patient. In future cases, the use of pharmacological functional MRI or PET scans with $N-\left[{ }^{11} \mathrm{C}\right]$-methyl-4-piperidyl acetate to measure acetylcholinesterase activity could be helpful in elucidating the pathophysiology of irinotecan-induced isolated dysarthria $[17,18]$.

In conclusion, selective irinotecan-induced dysarthria is an unusual and impressive entity, which can occur within minutes of irinotecan infusion. It is potentially related to a relative cholinergic oversensitivity of neurons in the area of the nucleus of the hypoglossal nerve. Though atropine can decrease cholinergic symptoms, it has not been conclusively shown to prevent the occurrence of dysarthria. Of note, as symptoms were fully reversible in all cases reported to date (in a period differing from $15 \mathrm{~min}$ [9] to several hours [this case, 12,13]) and did not increase in severity upon readministration 
of irinotecan, awareness of this phenomenon is critical in order to prevent unnecessary cessation of a potentially effective anticancer chemotherapeutic agent.
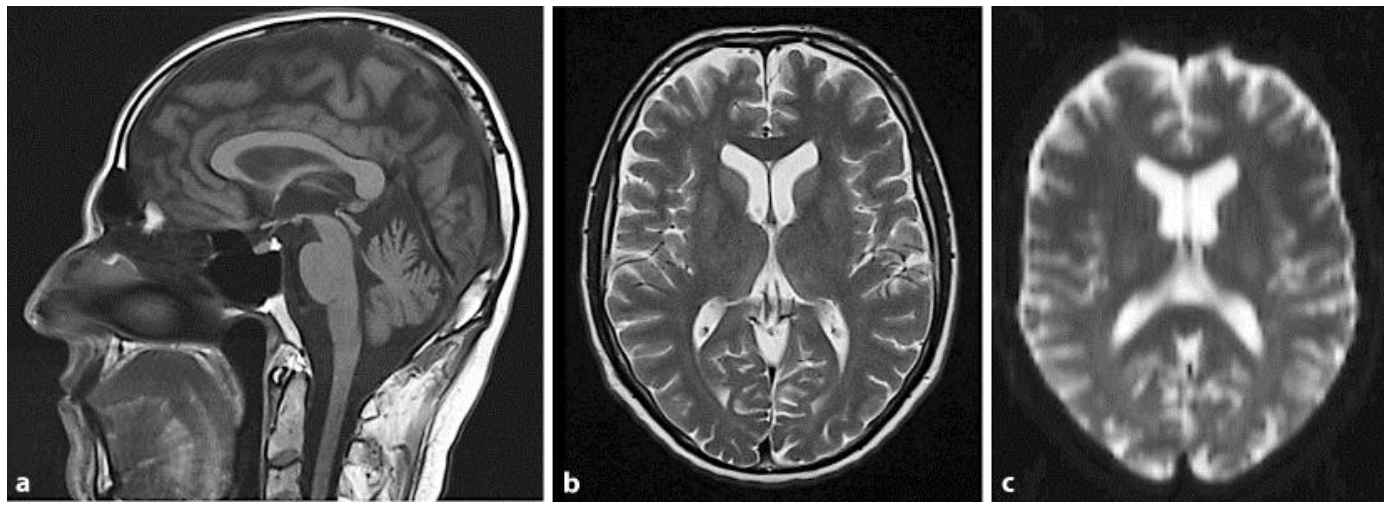

Fig. 1. Normal magnetic resonance imaging $(\mathbf{a}, \mathbf{b})$ and diffusion-weighted magnetic resonance imaging (c) of the brain during an episode of irinotecan-induced dysarthria.
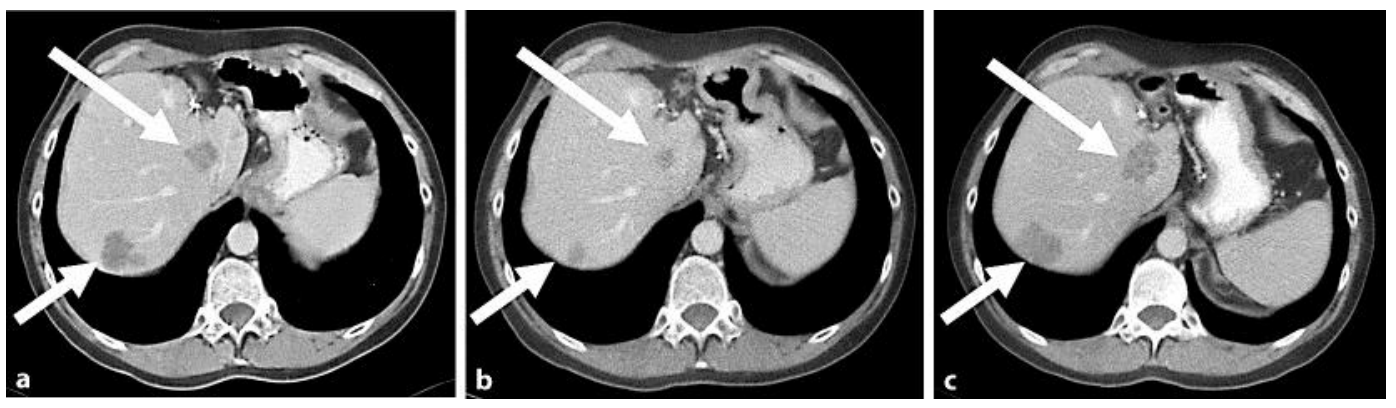

Fig. 2. Abdominal CT scans showing liver metastases (arrows) before irinotecan (a), after 6 cycles of irinotecan (b), and after 9 cycles of irinotecan (c).

\section{References}

1 Van Cutsem E, Twelves C, Cassidy J, et al: Oral capecitabine compared with intravenous fluorouracil plus leucovorin in patients with metastatic colorectal cancer: results of a large phase III study. J Clin Oncol 2001;19:4097-4106.

-2 Hoff PM, Ansari R, Batist G, et al: Comparison of oral capecitabine versus intravenous fluorouracil plus leucovorin as first-line treatment in 605 patients with metastatic colorectal cancer: results of a randomized phase III study. J Clin Oncol 2001;19:2282-2292.

-3 Punt CJ: Chemotherapy of patients with colorectal carcinoma. Ned Tijdschr Geneeskd 2005;149:14411447.

-4 Hurwitz H, Fehrenbacher L, Novotny W, et al: Bevacizumab plus irinotecan, fluorouracil, and leucovorin for metastatic colorectal cancer. N Engl J Med 2004;350:2335-2342.

5 Walko CM, Lindley C: Capecitabine: a review. Clin Ther 2005;27:23-44. 
6 Cassidy J, Clarke S, Díaz-Rubio E, et al: Randomized phase III study of capecitabine plus oxaliplatin compared with fluorouracil/folinic acid plus oxaliplatin as first-line therapy for metastatic colorectal cancer. J Clin Oncol 2008;26:2006-2012.

7 Harel M, Hyatt JL, Brumshtein B, et al: The crystal structure of the complex of the anticancer prodrug 7ethyl-10-[4-(1-piperidino)-1piperidino]-carbonyloxycamptothecin (CPT-11) with Torpedo californica acetylcholinesterase provides a molecular explanation for its cholinergic action. Mol Pharmacol 2005;67:1874-1881.

-8 Gomez JA, Sanchez I, Ramirez JA: Irinotecan-induced dysthria: an insight into its pathognesis? Gastrointestinal Cancer Res 2008;2:209-210.

$\checkmark 9$ De Marco S, Squilloni E, Vigna L, et al: Irinotecan chemotherapy associated with transient dysarthria and aphasis. Ann Oncol 2004;15:1147-1148.

10 Baz DV, Bofill JS, Nogueira JA: Irinotecan-induced dysarthria. J Natl Cancer Inst 2001;93:1419-1420.

11 Hamberg P, Donders RC, Ten Bokkel Huinink D: Central nervous system toxicity induced by irinotecan. J Natl Cancer Inst 2006;98:219.

-12 Hamberg P, Jong de FA, Brandsma D, et al: Irinotecan-induced central nervous system toxicity. Report on two cases and review of literature. Acta Oncol 2008;47:974-978.

13 Sevilla Garcia I, Rueda A, Alba E: Irinotecan-induced central nervous system toxicity: a case report. J Natl Cancer Inst 1999;91:647.

14 Love RJ, Webb WG: Neurology for the speech-pathologist, 4th edn, Boston, MA, Butterworth-Heinemann, 2001.

15 Cortes R, Probst A, Palacios JM: Quantitative light microscopic autoradiographic localization of cholinergic muscarinic receptors in the human brain: brainstem. Neuroscience 1984;12:1003-1026.

16 Haxhiu MA, Mitra J, van Lunteren E, et al: Hypoglossal and phrenic responses to cholinergic agents applied to ventral medullary surface. Am J Physiol Regulatory Integrative Comp Physiol 1984;247:939944.

17 Goekoop R, Scheltens P, Barkhof F, et al: Cholinergic challenge in Alzheimer patients and mild cognitive impairment differentially affects hippocampal activation - a pharmacological fMRI study. Brain 2006;129:141-157.

18 Shimada H, Hirano S, Shinotoh $\mathrm{H}$, et al: Mapping of brain acetylcholinesterase alterations in Lewy body disease by PET. Neurology 2009;73:273-278. 